\title{
Timely diagnosis of vascular Ehlers Danlos syndrome - the need for genetic testing and counselling
}

\author{
C Feben, ${ }^{1} \mathrm{~J}$ Kromberg, ${ }^{2}$ A McDonald, ${ }^{3}$ A Krause ${ }^{1}$ \\ ${ }^{1}$ Division of Human Genetics, National Health Laboratory Service \& School of Pathology, Faculty of Health Sciences, \\ The University of the Witwatersrand \\ ${ }^{2}$ Division of Human Genetics, School of Pathology, The University of the Witwatersrand \\ ${ }^{3}$ Alberts Cellular Therapy, Netcare Pretoria East Hospital
}

Corresponding author: Candice Feben (candice.feben@nhls.ac.za)

\begin{abstract}
Summary: We present a case of a young female patient diagnosed clinically and molecularly with V-EDS and briefly review the published literature on this severe condition. Additionally, the case is used to highlight the need for early confirmation of diagnosis and appropriate referral to allow surveillance, intervention and genetic counselling.
\end{abstract}

\section{Case Report}

A 24-year-old Caucasian woman, presented to a Genetic Clinic in Johannesburg, South Africa, referred from a haematologist who was concerned about her history of easy bruising and poor wound healing. Haematological causes for the bleeding diathesis had been excluded. She gave a history of easy and severe bruising, despite minimal trauma, starting in childhood. She reported ligament tears around both ankles after minor sporting accidents and a large spontaneous forearm haematoma. She also reported poor wound healing with abnormal scar formation particularly after surgery to insert a pin for a broken arm. She gave a history of a massive swelling of her neck (likely spontaneous haematoma) and a spontaneous compartment syndrome of her right calf. Neither incident was associated with trauma. She is one of a dizygotic pair. Her twin brother was clinically unaffected and there was no other significant family history.

Examination revealed a normally grown, non-dysmorphic female (apart from deep set eyes and asymmetry of the nares with the right smaller than the left). She was noted to have numerous bruises in various stages of healing as well as abnormal thin and puckered scars over her knees and right elbow. The scar from the operative repair of the right arm was thin and stretched. A large healing scar was noted over the lateral aspect of the right calf following treatment of the compartment syndrome. Her skin was fair with easily visible vasculature but no increased elasticity. A large varicose vein was noted over her left calf. Her joint hypermobility Beighton score $^{1}$ was 6/9 (Table 1). Echocardiography revealed significant regurgitant lesions of the mitral, tricuspid and aortic valves. She did not have scoliosis, pes planus/cavus or a pectus deformity. Examination of the chest, abdomen and spine was unremarkable.

A connective tissue disorder was suspected, particularly vascular Ehlers-Danlos syndrome ((V-EDS). The clinical phenotype met criteria for this condition as indicated in Table 2. As molecular testing for COL $3 A 1$ mutations was not available in South Africa at the time, a DNA sample was sent to a collaborating laboratory in Belgium (Centrum Medische Genetica; University of Antwerp). A thoracic aortic aneurysm and dissection genetic panel (TAAD panel) was performed. This next generation sequencing panel performs parallel and simultaneous sequencing of a number of genes associated with connective tissue disorders, including COL 3 A1. A heterozygous $C O L 3 A 1$ variant in intron $36(\mathrm{c} .2553+5 \mathrm{G}>\mathrm{A})$ was detected and was classified as a class 4 (probably pathogenic) splice site variant. The result strongly supported the clinical diagnosis of V-EDS in this woman.

Genetic counselling was provided for the patient and her family. Although it is most likely that her COL $3 \mathrm{Al}$ mutation arose de novo (in the absence of a family history consistent with an autosomal dominant pattern of inheritance) her twin brother was offered pre-symptomatic genetic testing as germline mosaicism in one of their parents could not be excluded. She was advised of her options regarding prenatal and pre-implantation genetic diagnosis given that she has a $50 \%$ risk of having an affected child. Referral was made to a vascular and cardiothoracic surgeon and appropriate surveillance, including a baseline magnetic resonance angiography (MRA), was recommended. 
Table 1: The Beighton Score of Joint Hypermobility ${ }^{1}$

\begin{tabular}{lcc}
\hline Clinical Feature & Score* & Patients Score \\
\hline $\begin{array}{l}\text { Passive extension of } 5^{\text {th }} \text { digit beyond } 90^{\circ} \\
\text { Passive flexion of thumb to volar aspect of the forearm }\end{array}$ & 1 point for each side & 2 \\
Passive hyperextension of the elbow joint beyond $180^{\circ}$ & 1 point for each side & 2 \\
Passive hyperextension of the knee joint beyond $180^{\circ}$ & 1 point for each side & 2 \\
$\begin{array}{l}\text { Forward flexion of the trunk with knees fully extended so that the palms of the hand rest } \\
\text { flat on the floor }\end{array}$ & 1 point \\
\hline Total & $\mathbf{9}$ points \\
\hline *A score of $\geq 5 / 9$ indicates generalized joint hypermobility & $\mathbf{6}$ \\
\hline
\end{tabular}

Table 2: Comparison of two Clinical Criteria systems for a clinical diagnosis of V-EDS *

\begin{tabular}{|c|c|c|c|}
\hline \multicolumn{2}{|l|}{ Major Criteria } & \multicolumn{2}{|l|}{ Minor Criteria } \\
\hline Villefranche $(1997)^{1}$ & International EDS Consortium $(2017)^{6}$ & Villefranch $(1997)^{1}$ & International EDS Consortium $(2017)^{6}$ \\
\hline $\begin{array}{l}\text { Arterial aneurysms, } \\
\text { dissection or rupture }\end{array}$ & Arterial rupture at a young age $(<40)$ & $\begin{array}{l}\text { Thin translucent skin } \\
\text { (especially noticeable on } \\
\text { chest/abdomen) }\end{array}$ & $\begin{array}{l}\text { Thin translucent skin with increased } \\
\text { venous visibility }\end{array}$ \\
\hline Intestinal rupture & $\begin{array}{l}\text { Spontaneous sigmoid colon perforation in } \\
\text { the absence of known diverticular disease } \\
\text { or other bowel pathology }\end{array}$ & $\begin{array}{r}\text { Characteristic facial ap } \\
\text { micrognathia, } \mathrm{n}\end{array}$ & $\begin{array}{l}\text { arance (thin vermillion of the lips, } \\
\text { row nose, prominent eyes) }\end{array}$ \\
\hline \multirow[t]{8}{*}{$\begin{array}{l}\text { Uterine rupture during } \\
\text { pregnancy }\end{array}$} & $\begin{array}{l}\text { Uterine rupture during the third trimester } \\
\text { of pregnancy in the absence of a } \\
\text { previous Caesarean section and/or severe } \\
\text { peripartum perineum tearing }\end{array}$ & Acrogeria (aged & pearance to the extremities) \\
\hline & \multirow[t]{7}{*}{$\begin{array}{l}\text { Carotid cavernous sinus fistula formation } \\
\text { in the absence of trauma }\end{array}$} & \multicolumn{2}{|c|}{$\begin{array}{l}\text { Hypermobility of small joints } \\
\text { Tendon/muscle rupture }\end{array}$} \\
\hline & & Early onset varicose veins & $\begin{array}{l}\text { Early onset varicose veins ( }<30 \text { years } \\
\text { and nulliparous if female) }\end{array}$ \\
\hline & & $\begin{array}{l}\text { Pneumothorax/ } \\
\text { haemopneumothorax }\end{array}$ & Spontaneous pneumothorax \\
\hline & & $\begin{array}{l}\text { Easy bruising (minimal } \\
\text { trauma/spontaneous) }\end{array}$ & $\begin{array}{l}\text { Bruising unrelated to identified } \\
\text { trauma and/or in unusual sites } \\
\text { (cheeks/back) }\end{array}$ \\
\hline & & \multicolumn{2}{|c|}{$\begin{array}{l}\text { Congenital dislocation of the hips } \\
\text { Talipes equinovarus }\end{array}$} \\
\hline & & Gingival recession & $\begin{array}{l}\text { Gingival recession or gingival } \\
\text { fragility }\end{array}$ \\
\hline & & $\begin{array}{l}\text { Chronic joint subluxations/ } \\
\text { dislocations } \\
\text { Carotid-cavernous sinus } \\
\text { arterio-venous fistula }\end{array}$ & Keratoconus \\
\hline
\end{tabular}

Based on the records of patients with V-EDS who have attended the Genetic Clinic in Johannesburg, South Africa, over the last 10 years, this woman is only the second patient known to have had molecular confirmation of her diagnosis. The other patient is currently 48 years old and has had a number of serious vascular incidents, the most significant of which resulted in right hemiplegia secondary to spontaneous left carotid artery dissection. Her COL3A1 mutation is a missense variant (G547R) which results in the substitution of arginine for glycine in the pro-collagen molecule.

\section{Discussion}

Vascular Ehlers-Danlos syndrome (V-EDS), previously known as type IV EDS, is a rare genetic condition characterised by abnormal structure and function of type III pro-collagen 
molecules and which manifests clinically with spontaneous medium and large size arterial rupture in early adulthood., ${ }^{1,2}$ The condition has an estimated worldwide prevalence of $1 / 50000-1 / 200000^{3}$. Severe vascular abnormalities, including aneurysm formation and spontaneous vessel rupture and dissection, often lead to serious complications depending on the artery involved. ${ }^{2} \mathrm{Up}$ to $65 \%$ of vascular events in affected individuals involve the medium or large arteries of the chest and abdomen, including the coronary arteries. Individuals may present with symptoms of a myocardial infarction, angina or abdominal pain. Seventeen percent of arterial events involve the head and neck region and manifest as cerebrovascular accidents or neurological abnormalities with the arms and legs being involved in a similar percentage. ${ }^{2}$ The average age of the first severe arterial event is estimated at 30-35 years of age. ${ }^{4}$ One quarter of affected individuals will have experienced a severe arterial complication by the age of 20 years; $90 \%$ by the age of 40 years. ${ }^{5}$ Massive arterial ruptures or dissections can lead to early death (average age of 50 years, slightly older in female patients). ${ }^{2,45}$ Affected individuals are also at risk for spontaneous gastrointestinal perforations, hollow organ rupture, spontaneous pneumo-haemothoraces, keratoconus and gingival recession. ${ }^{1,2}$ The hallmarks of the classic EDS phenotype, such as joint hypermobility, skin hyper-elasticity, inguinal and umbilical hernias and organ prolapse are variably present, but are not necessary for the diagnosis to be made. ${ }^{2,4}$ Consensus clinical diagnostic criteria were established in Villefranche in $1997^{1}$ and have most recently been updated by the International Ehlers-Danlos Consortium in $2017^{6}$ (Table 2 ). If one or more major criteria or several minor criteria are present, the diagnosis should be considered and confirmatory molecular genetic testing undertaken. ${ }^{2,46}$ It is important to note that the diagnostic criteria are specific in their need for molecular genetic analysis as the means of confirming a diagnosis of V-EDS. ${ }^{6}$ This highlights the need for the improvement of and access to genetic services, specifically in our local context.

V-EDS is an autosomal dominant genetic condition. The causative genetic mutation is inherited from an affected parent in $50 \%$ of cases and occurs as a de novo event in the other $50 \%{ }^{2}$ The mutation spectrum includes nonsense, missense and splice site variants, many of which result in substitution of other amino acids for glycine residues in the Gly-X-Y triplets of the major triple helical domain of the collagen protein. ${ }^{2,4}$ More than 600 variants have been described. ${ }^{2}$ Sequence analysis of COL3A1 identifies more than 95-98\% of pathogenic variants with deletion/duplication analysis predicted to pick up an additional $1 \%$ of cases., ${ }^{2,3}$ The mutation type has been shown to influence the disease severity, expected lifespan and incidence of certain complications including bowel perforation. ${ }^{4}$ In this regard, it is important to consider molecular confirmation of the diagnosis even when the clinical features are suggestive. In the event that mutation analysis for COL $3 \mathrm{Almutations}$ is negative in a patient with a clinically suggestive phenotype, mutation analysis of COL1A1should be considered as rare cases of the V-EDS phenotype have been ascribed to mutations in this gene. ${ }^{6}$ Alternatively, next generation sequencing panels which simultaneously assess a number of genes implicated in connective tissue pathology can be used as a first-line testing strategy. ${ }^{6}$

As there is a $50 \%$ risk of an affected individual passing the mutation to their offspring (even if the proband is the first affected individual in a family), various options exist for both prenatal and pre-implantation genetic diagnosis once the causative genetic mutation has been identified. ${ }^{2}$ Although pregnancies are not contraindicated, there is a $15 \%$ risk for life-threatening complications to occur during a pregnancy or delivery in affected females. ${ }^{2}$ Presymptomatic testing of minors is considered appropriate given the age of onset of symptoms and the possibility of serious complications before the age of 18 years. ${ }^{4}$

Given the severity of the condition, certain surveillance and management measures should be put in place as soon as a diagnosis is suspected. The creation of a care team which should include the medical geneticist, general surgeon, vascular surgeon, cardiologist and physician, is strongly recommended. ${ }^{3}$ Although guidelines will vary according to location and the availability of expertise, it has been suggested that initial MRA, with venous contrast should be performed to identify internal aneurysms and arterio-venous fistulae. ${ }^{3}$ These may be amenable to endovascular or open repair prior to the development of complications. ${ }^{2,3}$ The frequency of repeat imaging should ideally be on an annual basis if feasible ${ }^{3}$ or can be determined by the findings of the initial scans. ${ }^{2}$ Surgical complications can include wound dehiscence, bleeding at fragile tissue sites, fistula formation, infections and the need for repeat intervention. ${ }^{4}$ Beta-blocker therapy, specifically with celiprolol, has been shown to reduce the overall frequency of arterial ruptures and dissections and should be considered in all cases. ${ }^{5}$

Additionally, it is recommended that affected individuals avoid contact sports, weight lifting or weight training. Moderate recreational activity is not contraindicated. Routine colonoscopy should be avoided in view of the risk of gastrointestinal perforation and elective surgery should be discouraged unless the expected benefit is substantial. The use of medic alert bracelets is also strongly advised. ${ }^{2}$

The diagnosis of V-EDS has potentially devastating consequences - the personal and familial impact of the disorder needs to be carefully discussed with the affected individual and their at-risk family members and, in this regard, genetic counselling is recommended., ${ }^{2,3}$

\section{Conclusion}

V-EDS is a rare, although severe, autosomal dominant connective tissue disorder. Prompt recognition of the condition, confirmation of the clinical diagnosis, appropriate referral to a medical geneticist and vascular surgeon may all assist in reducing the potentially life-threatening complications of this condition. 


\section{Acknowledgements}

We would like to thank the patients and their families for their participation and permission to use their information in the drafting of this report. We would also like to thank Professor Bart Loeys from the University of Antwerp, Belgium, for his assistance with the genetic testing for this patient. This report was approved by the Human Research Ethics Committee of the University of the Witwatersrand.

\section{REFERENCES}

1. Beighton P, De Paepe A, Steinmann B, et al. Ehlers-Danlos syndromes: revised nosology, Villefranche, 1997. EhlersDanlos National Foundation (USA) and Ehlers-Danlos Support Group (UK). Am J Med Genet. 1998;77:31-7.
2. Pepin MG, Murray ML, Byers PH. Vascular Ehlers Danlos Syndrome. Gene Reviews. 2015. Available at: www.ncbi.nlm. nih.gov/books/NBK1494/

3. Byers PH, Belmont J, Black J, et al. Diagnosis, natural history, and management in vascular Ehlers-Danlos syndrome. Am J Med Genet C Semin Med Genet. 2017;175C:40-7.

4. Pepin MG, Schwarze U, Rice KM, et al. Survival is affected by mutation type and molecular mechanism in vascular EhlersDanlos syndrome (EDS type IV). Genet Med. 2014;16: 881-8.

5. Ong KT, Perdu J, De Backer J, et al. Effect of celiprolol on prevention of cardiovascular events in vascular Ehlers-Danlos syndrome: a prospective randomised, open, blinded-endpoints trial. Lancet. 2010;376:1476-84.

6. Malfait F, Francomano C, Byers PH, et al. The 2017 International Classification of the Ehlers-Danlos Syndromes. Am J Med Genet C Semin Med Genet. 2017;175C:8-26. 